\title{
Enhancement of breast periphery region in digital mammography
}

\section{Ana Luiza Menegatti Pavan, Antoine Vacavant, Andre Petean Trindade, Caio Cesar Quini, Diana Rodrigues de Pina}

Ana Luiza Menegatti Pavan, Antoine Vacavant, Andre Petean Trindade, Caio Cesar Quini, Diana Rodrigues de Pina, "Enhancement of breast periphery region in digital mammography," Proc. SPIE 10574, Medical Imaging 2018: Image Processing, 105742R (2 March 2018); doi: $10.1117 / 12.2293547$

SPIE. Event: SPIE Medical Imaging, 2018, Houston, Texas, United States 


\title{
Enhancement of Breast Periphery Region in Digital Mammography
}

\author{
Ana Luiza Menegatti Pavan ${ }^{\mathrm{a}}$, Antoine Vacavant ${ }^{\mathrm{b}}$, Andre Petean Trindade ${ }^{\mathrm{c}}$, Caio Cesar Quini ${ }^{\mathrm{a}}$, Diana \\ Rodrigues de Pina*c \\ ${ }^{a}$ Dept of Physics and Biophysics, Biosciences Institute of Botucatu, São Paulo State University, \\ Distrito de Rubião Junior S/N, Botucatu, São Paulo, 18618-000. Brazil; bept. Institut Pascal, \\ Université Clermont Auvergne, UMR 6602 UCA/SIGMA/CNRS, F-63171 Aubière, France, ${ }^{\mathrm{c} D e p t}$ \\ of Tropical Diseases and Diagnostic Imaging, Botucatu Medical School, Univ Estadual Paulista, \\ Distrito de Rubião Junior S/N, Botucatu, São Paulo, 18618-000, Brazil.
}

\begin{abstract}
Volumetric breast density has been shown to be one of the strongest risk factor for breast cancer diagnosis. This metric can be estimated using digital mammograms. During mammography acquisition, breast is compressed and part of it loses contact with the paddle, resulting in an uncompressed region in periphery with thickness variation. Therefore, reliable density estimation in the breast periphery region is a problem, which affects the accuracy of volumetric breast density measurement. The aim of this study was to enhance breast periphery to solve the problem of thickness variation. Herein, we present an automatic algorithm to correct breast periphery thickness without changing pixel value from internal breast region. The correction pixel values from periphery was based on mean values over iso-distance lines from the breast skin-line using only adipose tissue information. The algorithm detects automatically the periphery region where thickness should be corrected. A correction factor was applied in breast periphery image to enhance the region. We also compare our contribution with two other algorithms from state-of-the-art, and we show its accuracy by means of different quality measures. Experienced radiologists subjectively evaluated resulting images from the tree methods in relation to original mammogram. The mean pixel value, skewness and kurtosis from histogram of the three methods were used as comparison metric. As a result, the methodology presented herein showed to be a good approach to be performed before calculating volumetric breast density.
\end{abstract}

Keywords: Mammography, peripheral enhancement, thickness correction, image processing

\section{INTRODUCTION}

Estimation of volumetric breast density (VBD) has been widely discussed in the recent literature [1-7]. Fibroglandular tissue in the breast is represented as radiographically dense tissue on a mammogram [8-10]. VBD has been shown to be one of the strongest risk factors for breast cancer diagnosis $[4,8,9]$, which is being used to personalize preventive and screening strategies for women $[4,11,12]$. Women with high breast density have increased risk of developing breast cancer in twofold to sixfold compared to women with low breast density $[5,8,13,14]$.

Digital mammography introduction has made feasible to develop automated volumetric methods $[5,15,16]$. To do so, accurate knowledge of tissue thickness at each pixel in mammogram is necessary $[5,7,16]$. However, volumetric methods are highly sensitive to the breast thickness [16-18]. This is particularly difficult to estimate at the breast periphery $[5,19]$. During mammography acquisition, breast is compressed and part of it loses contact with the paddle [7, 17], resulting in an uncompressed region in periphery with thickness variation. Differences in pixel intensities in periphery are therefore governed principally by breast thickness $[5,18]$.

Procedures to correct the non-uniform tissue thickness in mammograms are necessary to accurately estimate VBD [13]. Pre-processing techniques should be used in mammograms to enhance peripheral areas visibility and improve intensity distribution [20]. Studies show the development of algorithms to identify the peripheral region on mammogram and to correct overexposed area $[13,17,21,22]$. In the literature this approach has been called as thickness equalization or peripheral enhancement [22].

Medical Imaging 2018: Image Processing, edited by Elsa D. Angelini, Bennett A. Landman, Proc. of SPIE Vol. 10574, 105742R · (C) 2018 SPIE · CCC code: 1605-7422/18/\$18 - doi: 10.1117/12.2293547 
The different methods realizing breast thickness periphery correction may vary in the determination of the correction area and in the used correction factor. Regarding area, algorithms can be either applied to the whole image [13, 23, 24] or just to a region determined by a segmentation algorithm [20-22].

In the first case, as Wang et al. method [13], the fitted enhancement curve defines the necessary correction value for each pixel, which is added to the original pixel value to create the processed image. Such an approach applies the thickness correction to the entire breast. It works well with a homogeneous fatty and dense breast but localized artefacts can be seen when a breast exhibits large density variations across the mammogram [20,21]. As a consequence, these methods may be unappropriated to be used before the VBD measurement. This fact is due to the pixel values of entire breast are altered, even where there is no overexposed problem, which influences in three-dimensional information.

In the second case, as Tortajada et al. [21] method, the breast thickness correction is iteratively applied in a region determined by a segmentation algorithm. These methods segment the breast periphery and calculate the correction factor based on periphery neighborhood. The approach works well with adipose and scattered fibroglandular breasts. However, breast with large dense regions near the periphery neighborhood may overestimate the correction factor in first iterations. Since breast periphery is a predominantly adipose region [25], correction factor overestimation may cause a bias in future VBD estimation.

In this study, we present an algorithm that corrects intensities variations in breast periphery using digital mammograms. The aim herein is to implement a new hybrid methodology, which could be used independent of breast size, breast composition and mammographic view. In Section B, we present the developed algorithm. We also compare, in Section $\mathrm{C}$, the results from corrected and uncorrected images with other methodologies of literature.

\section{METHODOLOGY}

\subsection{Dataset}

The present study was developed with ethical approval from the authors' institutions under protocol number 50547315.8 .0000 .5411 .

Women aged 18 years or older undergoing screening mammography between 2013 and 2015 at Botucatu Medical School were included. For a woman who has been included in the study, she must have had no previous history of breast cancer or breast surgery, and had a BI-RADS assessment of either 1 or 2 (negative or benign finding, respectively). Only mammography of one side, left or right, was used for each patient in craniocaudal (CC) view, allowing assessment of different breast compositions. Third mammograms were evaluated.

The computed radiography (CR) mammography system used was a Senographe 600T (GE Healthcare, Milwaukee, WI) with a CR-85X image digitizer (Agfa-Gevaert Group, Mortsel, BE). An Agfa image plate $(18 \times 24 \mathrm{~cm} 2)$ with a pixel pitch of $50 \mu \mathrm{m}$ was employed. A Mo/Mo anode/filter combination was used for all X-ray exposures. Processed images were used, since raw data is often not achieved in clinical practice [20].

\subsection{Developed Algorithm}

The proposed algorithm has been developed in Matlab software to enhance breast periphery region in a mammogram. The algorithm is a fully automatic hybrid method, which uses different image processing techniques. It is based on mean values over iso-distance lines from the breast skin-line. Detailed process is explained in the following subsections.

\section{Preprocessing}

In the first stage, a median filter is applied in the mammogram to reduce image noise and to allow good breast edge detection. Then, the mammogram is segmented into the breast area and the background by thresholding, resulting in a mask with the region of interest. 


\section{Adipose Tissue Otsu Thresholding}

To acquire an image with only adipose tissue, the fibroglandular tissue intensity level is removed from breast area by Otsu thresholding process [26]. To define the Otsu threshold, only the compressed breast area is employed, in order to avoid bias segmentation of pixels from periphery region. Therefore, the breast mask is eroded to estimate the compressed area, as highlighted in white in Fig.1a. We apply Otsu thresholding technique in the estimated compressed area to define a threshold value [8]. This value is then used to segment fibroglandular tissue in complete breast region, resulting in an image with only adipose tissue area. At this stage, the segmentation of dense tissue is roughly estimated, as shown in Fig. $1 b$.
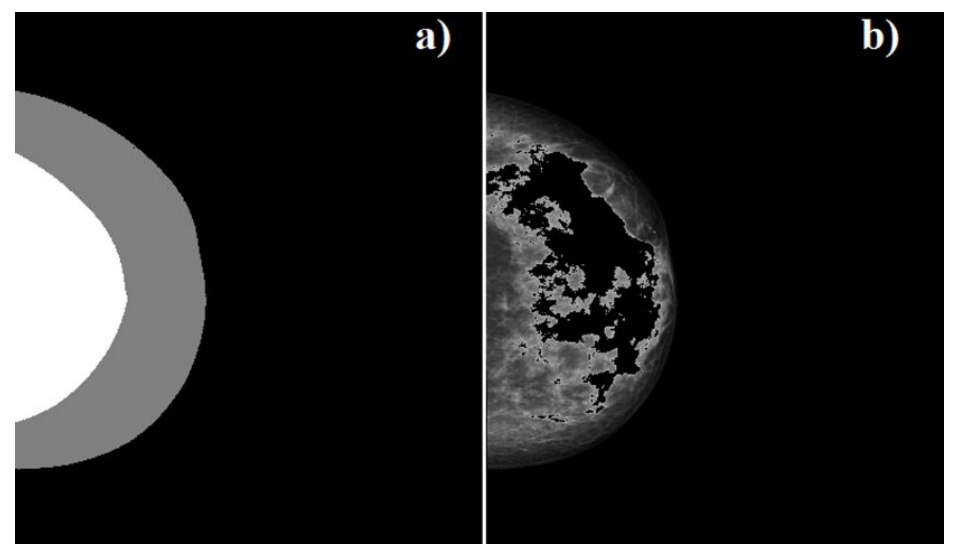

Figure 1. a) Rough estimation of compressed (white) and uncompressed (gray) breast area; b) Adipose tissue after segmentation of fibroglandular tissue by Otsu thresholding.

\section{Distance Mapping}

Using the adipose tissue area estimated before, a distance map is generated by calculating the shortest distance from each pixel to the skin-line. Each pixel from adipose tissue area is labeled with its minimum Euclidean distance to the skinline, as shown in Fig. 2a. Through the distance map, an intensity curve is created based on the average intensity level values that are within the same distances $(d)$ to the skin-line. Therefore, the curve is an approximation of the mean intensity values from pure adipose tissue in relation of $d$. Fig. 2b shows the intensity curve and its two-term exponential model fit $(M(d))$.
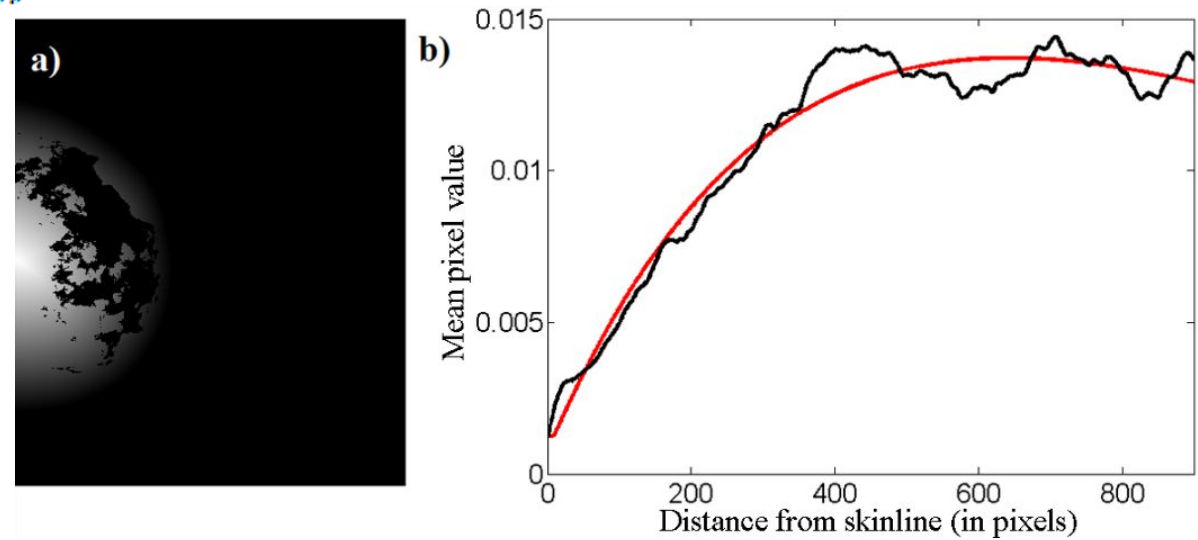

Figure 2. a) Distance map of adipose tissue; b) Intensity curve (black) and its fit (red) for the mean pixel values of adipose tissue.

\section{Identifying Periphey Region}

The exponential fit $\mathrm{M}(\mathrm{d})$ for adipose tissue area shows that the pixel value from periphery region strongly varies in relation with the distance from skin-line. On the other hand, compressed region has almost constant thickness and, 
therefore, intensity values vary slowly. To identify the distance from the skin-line that separates compressed to uncompressed areas, the algorithm calculates the fit's derivate. By the analysis of derivate, the optimum position, which defines the limit between both areas, is defined as the first point in which the derivate is less than 0.1, representing the beginning of a constant value in the fit. Fig. 3a shows the derivate and the selected point (p). Fig. 3b shows an example of segmentation with gray and white areas indicating the compressed and uncompressed regions respectively.

a)

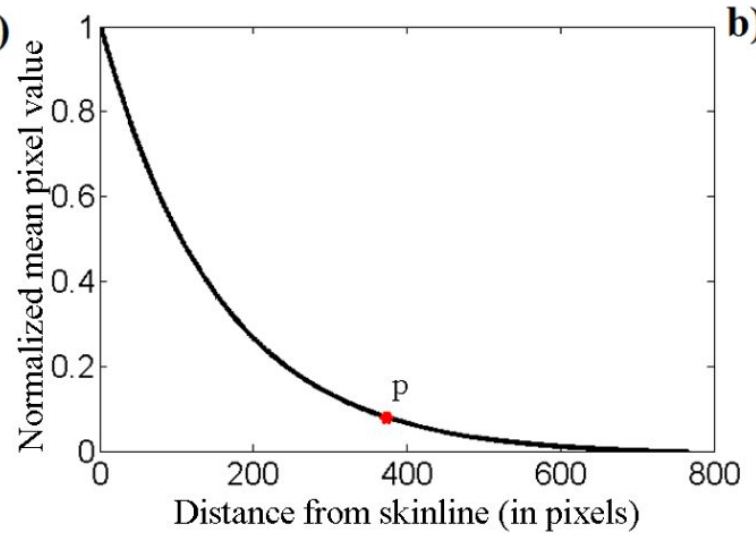

b)

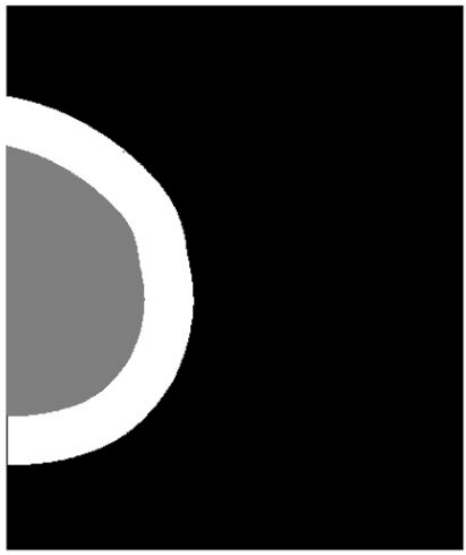

Figure 3. a) Fit's derivate and the point (p) which the derivate is approximately zero; b) Accurately compressed (gray) and uncompressed (white) segmentation regions.

\section{Correction Factor}

The periphery area is corrected by using a correction factor. For each distance $d$ within the breast area, a thickness correction factor $(C F)$ was calculated as in:

$$
C F(d)=\frac{M_{\max }}{M(d)}
$$

where $M_{\max }$ is the maximal value from $M(d)$.

The $C F$ is then multiplied by each pixel value from the periphery region, allowing enhancement of the area.

\section{Comparison with Other Methods}

For the same dataset, periphery enhancement is corrected using our method and compared with the ones proposed by Wang et al. [13] and He et al. [20], which used screen-film and full field digital mammography, respectively. Therefore, methods were adapted for CR mammograms. For He's method only breast periphery separation and intensity ratio propagation steps were used since our dataset is composed with only CC view. The histogram for images before and after enhancement were evaluated using the three methodologies. Skewness, kurtosis and mean pixel values were also used to compare methods. The values are presented as median and interquartile range. Since all data are nonparametric, we perform paired Wilcoxon signed-rank test to compare the different methods.

A relative visual grading analysis (VGA) was made by three experienced radiologists to compare image quality after periphery correction between the methodologies described in this paper and original mammogram. Radiologists gave a score for each corrected image varying from +2 to -2 to describe image quality in relation to periphery visualization [27, 28]. Positive score means an improvement in image quality, highlighting periphery visualization. Zero score represents that the correction did not change the periphery visualization relative to the original image, preserving original image quality. A negative score means a reduction of image quality when compared with original mammogram. In this case, 
even with better visualization of breast periphery, a global analysis of image quality, reveals to be worse than original one. A resulting score was achieved by summing all scores and dividing by the number of observers (radiologists) and the number of assessed images for each observer [27, 28]. For the purpose of this work, radiologists were not allowed to window image contrast.

\section{RESULTS}

Fig. 4 shows comparison between original (without periphery enhancement- Fig. 4a-c) and enhanced images by the proposed (Fig. 4d-f), Wang, et al. (Fig. 4g-i) and He, et al. (Fig. 4j-1) methods, respectively. Images are represented for the same window width and center contrast adjustments.
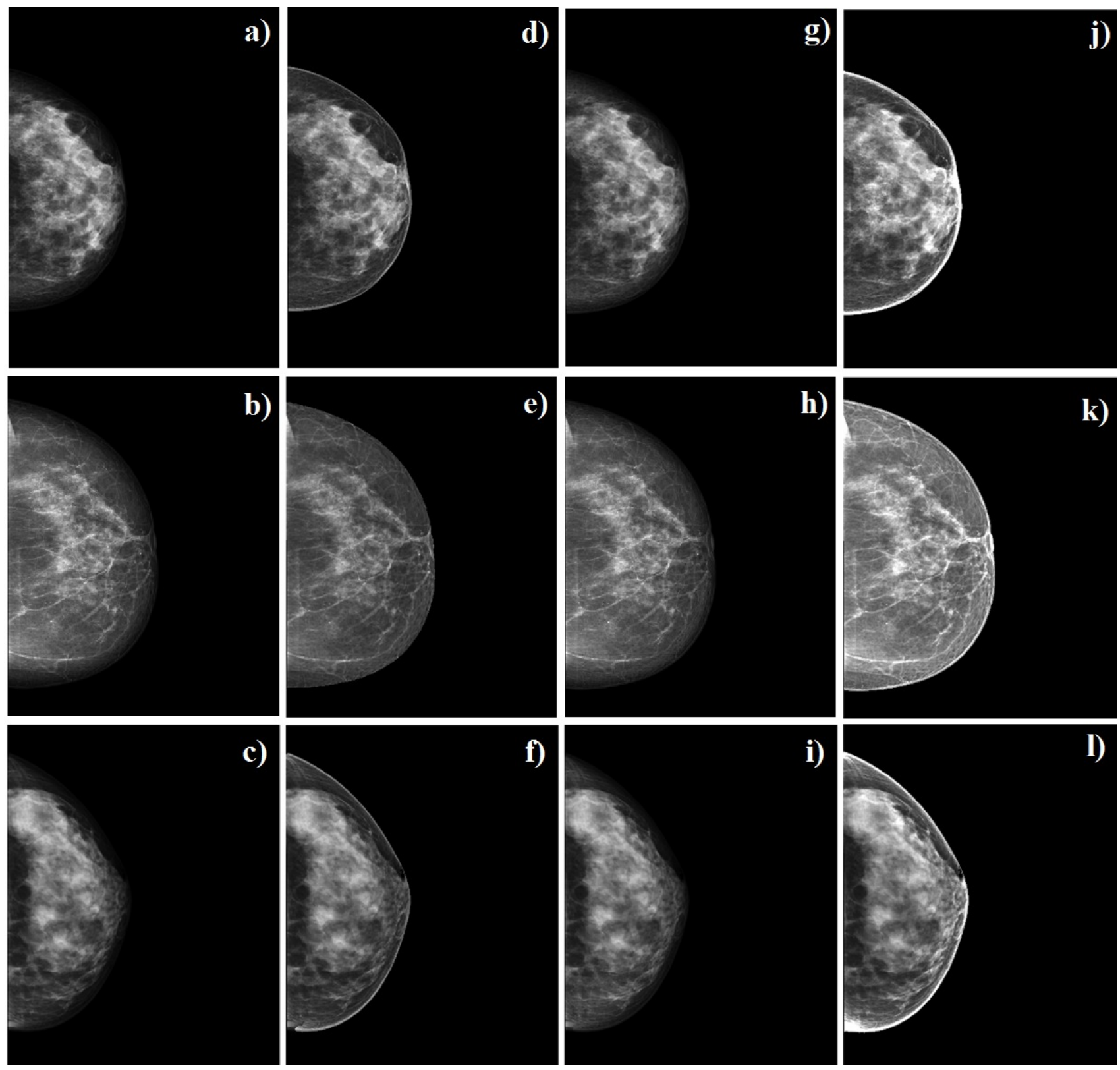

c)

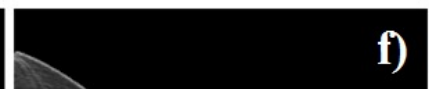

)

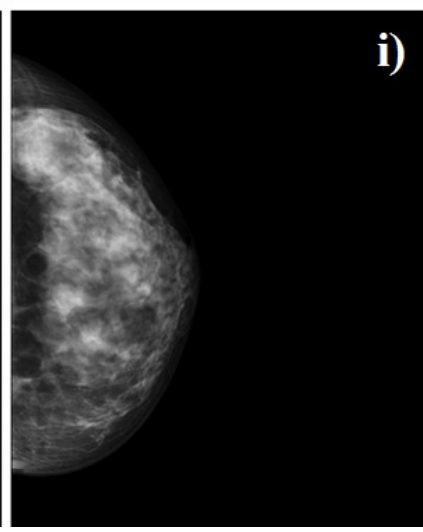

t.

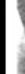

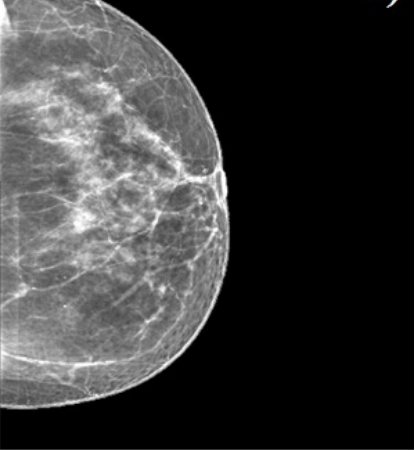

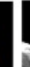

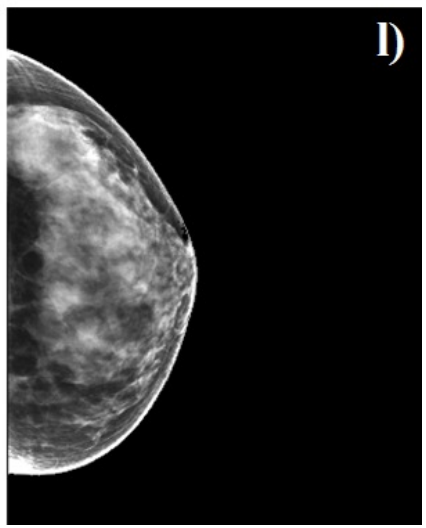

Figure 4. a-c) Original images; Breast periphery correction by d-f) the proposed, g-i) Wang, et al. and j-1) He, et al. methods. 
Fig. 5 shows examples of histograms from images of Fig. 4. In Fig. 5 d-f histograms for original mammogram (Fig. 4a-c) are represented by black line, for the proposed (Fig. 4d-f) are represented by red line, Wang et al. (Fig. 4g-i) are represented by blue line and $\mathrm{He}$ et al. (Fig. 4j-1) are represented by green line.
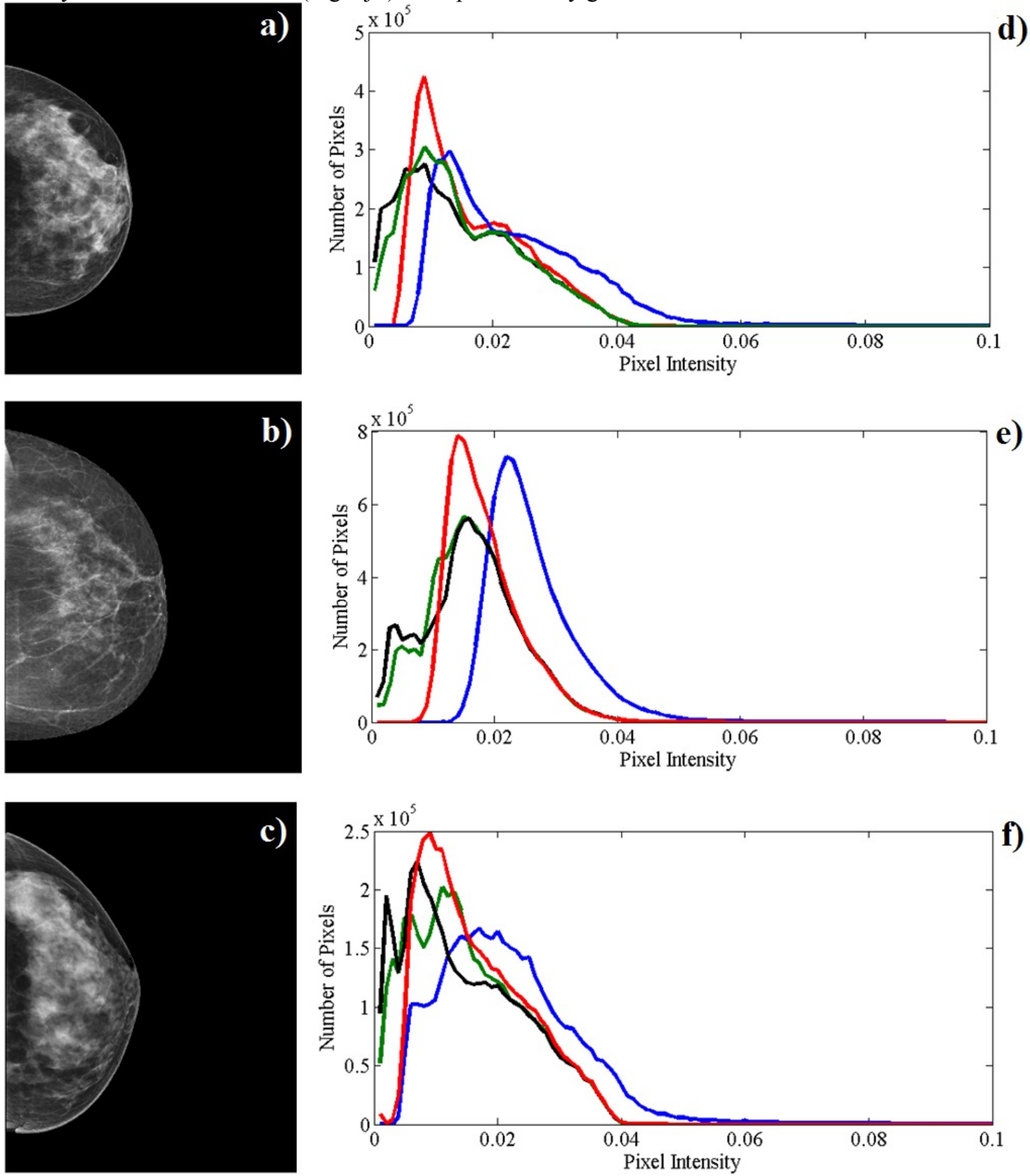

Figure 5. a-c) Example of images used to acquire histograms; d-f) Comparison of histograms between original image (black), proposed (red), Wang et al. (blue) and He et al. (green) methods.

Table 1 shows comparison between methods in relation of mean pixel value, skewness and kurtosis for 30 assessed mammograms. Different letters indicate statistical difference $(p<0.05)$. 
Table 1. Results of mean pixel value, skewness and kurtosis for the proposed, Wang and He methods.

\begin{tabular}{ccccc}
\hline & Original & Proposed Method & Wang & He \\
\hline $\begin{array}{c}\text { Mean pixel } \\
\text { value }\left(\mathbf{x 1 0}^{-3}\right)\end{array}$ & $15.67(14.88-16.68)^{\mathbf{a}}$ & $16.86(16.23-18.15)^{\mathbf{b}}$ & $24.02(23.11-29.98)^{\mathbf{c}}$ & $16.02(15.22-17.03)^{\mathbf{d}}$ \\
Skewness & $0.92(0.41-1.30)^{\mathbf{a}}$ & $1.41(1.05-1.79)^{\mathbf{b}}$ & $1.55(1.11-1.78)^{\mathbf{b}}$ & $0.99(0.51-1.50)^{\mathbf{a}}$ \\
Kurtosis & $2.80(2.12-3.86)^{\mathbf{a}}$ & $3.85(2.83-5.02)^{\mathbf{b}}$ & $4.04(3.03-4.82)^{\mathbf{b}}$ & $2.69(1.98-4.76)^{\mathbf{a}}$ \\
\hline
\end{tabular}

As a result of radiologists visual assessment, the final VGA score was $+1.5,-0.3$ and +0.7 for our, Wang and $\mathrm{He}$ methodologies respectively. All images had better visualization of periphery area when compared with original mammogram

\section{DISCUSSION}

We have developed a methodology for digital mammogram thickness periphery correction to be used in future work of VDB estimation. Our automatic approach combines different image processing techniques, such as morphological operators, thresholding and distance mapping, to define a valid correction factor. The correction factor is used to enhance breast periphery without changing pixel value of the compressed breast region.

Fig. 4 shows an example of original and enhanced images, which are realized by the proposed, Wang and He methods. By radiologists visual assessment all images had better visualization of periphery area when compared with original mammogram. Subjective examination of processed images by the proposed method (Fig. 4d-f) reveals a good enhanced image presentation with structural information remaining unaltered by the correction algorithms, resulting in a VGA score near to the maximum value. This is due to correction factor is applied only in breast periphery. Using the method of He et al. to compare with original mammograms, radiologists describe moderated improvement of peripheral area. Although images corrected by this method were slightly better, the correction factor was not sufficient to correct all periphery area resulting in a VGA score of 0.7 . This can be seen in Fig. 4g-i, since periphery area presents a gradient, resulting in lower correction near skin-line. In fibroglandular breasts cases, it is possible to notice intensity overestimation of pixels located close to frontier between compressed and uncompressed areas. By analyses of Fig. 4j-1, it can be seen that periphery is corrected. Nevertheless, radiologists reported a degradation of image contrast in area near to chest wall since pixels from compressed area were also multiplied by the correction factor resulting in higher intensity values. Therefore, although the periphery was better visualized in corrected image using Wang method, image quality from breast compressed area was worse, resulting in a negative VGA score of -0.3 .

Fig. 5 shows examples of histograms for evaluated images in Fig. 4, for original image and after periphery enhancement realized by the three methods. Comparing the histogram from proposed method with the original, we may notice that the profile of histograms has almost the same shape for high pixel values. Histogram from proposed method is condensed to a smaller range of intensity values. This is due to pixels from periphery breast region (i.e. overexposed) that have been gathered to a central part of histogram after correction. Therefore, overexposed pixels are reallocated obtaining a more adipose homogeneous intensity distribution. Furthermore, the maximum pixel value of histogram was maintained. The histogram's shape from processed image by He method is also quite similar to the original. However, there are still low intensity pixels, which were not correctly corrected. This occurs due to the used correction factor losing power each iteration and, therefore, pixels near skin-line are multiplied by a low correction factor. Blue lines of Fig. 5 shows that the entire histograms are displaced to the right when using the Wang correction method. This modifies both the shape and the maximum pixel value of histogram.

Table I shows that the phenomenon illustrated in Fig. 5 is maintained in all assessed images. For the mean pixel value, all methods are statically different. However, for the proposed and He methods, the mean pixel value has a proximate value from the original (around $7 \%$ and $2 \%$ greater than the original value, respectively) because only periphery area is corrected. Wang method has a higher mean value (around 50\% greater than the original value), due to its use of a correction factor in the entire breast region, not only in the periphery area. 
Analyzing skewness and kurtosis values, the Table I shows that proposed and Wang methods are significantly different from original value. However, no differences were found between them. He method showed no differences when compared with original image.

The skewness measures how asymmetric the histogram is, 0 being a totally symmetric distribution [21]. Negative or positive values for slope indicate histograms that are inclined to the left or to the right, respectively. The presented skewness values agree with observed behavior in Fig. 5. For proposed and Wang methods, the median skewness is greater than the original value, which is due to the histogram of the processed image to be slightly skewed to the right. For the Wang method, the median skewness is higher, since all pixels in the image are corrected, inclining the histogram on the right.

The kurtosis measures how wide the peak is. Positive or negative kurtosis indicates a "heavy-tailed" or "light-tailed" distribution, respectively [21]. The presented kurtosis values from Table I also agree with observed behavior in Fig. 5. For proposed and Wang methods, the median kurtosis is greater than the original value, which is due to the histogram of the processed image to present a greater number of pixels in a smaller range of intensity values, i.e. a wide peak.

Skewness and kurtosis using He method are similar to the original image, because of pixels near to skin-line were not efficiency enhanced resulting in subtle changes in histogram.

Although the proposed method presents some advantages over the ones in literature, the aim of the work was to compare it with widely used methods, since no ground truth is available. We have shown that the proposed method is accurate and robust to automatically perform the enhancement of periphery breast region. The method presented herein fits enhancement curve based exclusively on adipose tissue of distance map, since the adipose tissue in compressed region is basically homogeneous and variations in the pixel values of periphery are governed by the breast thickness [5]. The fit is used to calculate the correction factor, which is applied in each pixel of uncompressed region. Then, the method is effective even on very dense breasts. Furthermore, the use of distance map allows to speed-up the correction of periphery breast region, since the use of it makes possible to deal with all pixels at the same distance at the same time [21].

\section{CONCLUSION}

As a conclusion, the methodology presented herein may be used independent of breast size, breast composition and mammographic view. Therefore, the proposed method shows to be a good approach to be performed before calculating $\mathrm{VBD}$, since it does not change the pixel values of the interior breast region.

\section{ACKNOWLEDGEMENTS}

The authors thank all of the clinical and technical personnel of our institution radiodiagnostic facility. The authors also thank the radiologists: Sergio Marrone Ribeiro, Andre Petean Trindade, and Seizo Yamashita. Financial support was provided by CNPq and CAPES under process PDSE $n^{\circ} 88881.132793 / 2016-01$. This work is a part of the project INCTFNA Proc. No. 464898/2014-5.

\section{REFERENCES}

[1] Alonzo-Proulx, O., Jong, R. A., and Yaffe, M. J., "Volumetric breast density characteristics as determined from digital mammograms," Phys Med Biol, 57(22), 7443-57 (2012).

[2] Wang, J., Azziz, A., Fan, B. et al., "Agreement of mammographic measures of volumetric breast density to MRI," PLoS One, 8(12), e81653 (2013).

[3] Gubern-Merida, A., Kallenberg, M., Platel, B. et al., "Volumetric breast density estimation from full-field digital mammograms: a validation study," PLoS One, 9(1), e85952 (2014). 
[4] Eng, A., Gallant, Z., Shepherd, J. et al., "Digital mammographic density and breast cancer risk: a case-control study of six alternative density assessment methods," Breast Cancer Res, 16(5), 439 (2014).

[5] Chen, X., Moschidis, E., Taylor, C. et al., [Improving Mammographic Density Estimation in the Breast Periphery.] Springer International Publishing, Malmö Live, Malmö, Sweden(2016).

[6] Keller, B. M., Nathan, D. L., Wang, Y. et al., "Estimation of breast percent density in raw and processed full field digital mammography images via adaptive fuzzy c-means clustering and support vector machine segmentation," Med Phys, 39(8), 4903-17 (2012).

[7] Holland, K., Gubern-Merida, A., Mann, R. M. et al., "Optimization of volumetric breast density estimation in digital mammograms," Phys Med Biol, 62(9), 3779-3797 (2017).

[8] Kallenberg, M. G., Lokate, M., van Gils, C. H. et al., "Automatic breast density segmentation: an integration of different approaches," Phys Med Biol, 56(9), 2715-29 (2011).

[9] Assi, V., Warwick, J., Cuzick, J. et al., "Clinical and epidemiological issues in mammographic density,” Nat Rev Clin Oncol, 9(1), 33-40 (2011).

[10] Winkler, N. S., Raza, S., Mackesy, M. et al., "Breast density: clinical implications and assessment methods," Radiographics, 35(2), 316-24 (2015).

[11] Schousboe, J. T., Kerlikowske, K., Loh, A. et al., "Personalizing mammography by breast density and other risk factors for breast cancer: analysis of health benefits and cost-effectiveness," Ann Intern Med, 155(1), 10-20 (2011).

[12] Checka, C. M., Chun, J. E., Schnabel, F. R. et al., "The relationship of mammographic density and age: implications for breast cancer screening," AJR Am J Roentgenol, 198(3), W292-5 (2012).

[13] Wang, X. H., Good, W. F., Chapman, B. E. et al., "Automated assessment of the composition of breast tissue revealed on tissue-thickness-corrected mammography,” AJR Am J Roentgenol, 180(1), 257-62 (2003).

[14] Li, J., Szekely, L., Eriksson, L. et al., "High-throughput mammographic-density measurement: a tool for risk prediction of breast cancer," Breast Cancer Res, 14(4), R114 (2012).

[15] Kallenberg, M. G., van Gils, C. H., Lokate, M. et al., "Effect of compression paddle tilt correction on volumetric breast density estimation," Phys Med Biol, 57(16), 5155-68 (2012).

[16] Yaffe, M. J., "Mammographic density. Measurement of mammographic density,” Breast Cancer Res, 10(3), 209 (2008).

[17] Alonzo-Proulx, O., Mainprize, J. G., Packard, N. J. et al., [Development of a Peripheral Thickness Estimation Method for Volumetric Breast Density Measurements in Mammography Using a 3D Finite Element Breast Model] Springer, Berlin, Heidelberg., Catalonia, Spain(2010).

[18] van Engeland, S., Snoeren, P. R., Huisman, H. et al., "Volumetric breast density estimation from full-field digital mammograms," IEEE Trans Med Imaging, 25(3), 273-82 (2006).

[19] Alonzo-Proulx, O., Packard, N., Boone, J. M. et al., "Validation of a method for measuring the volumetric breast density from digital mammograms," Phys Med Biol, 55(11), 3027-44 (2010).

[20] He, W., Hogg, P., Juette, A. et al., "Breast image pre-processing for mammographic tissue segmentation," Comput Biol Med, 67, 61-73 (2015).

[21] Tortajada, M., Oliver, A., Marti, R. et al., "Breast peripheral area correction in digital mammograms," Comput Biol Med, 50, 32-40 (2014).

[22] Snoeren, P., and Karssemeijer, N., "Thickness correction of mammographic images by means of a global parameter model of the compressed breast.," IEEE Trans Med Imaging, 23(7), 799-806 (2004).

[23] Bick, U., Giger, M. L., Schmidt, R. A. et al., "Density correction of peripheral breast tissue on digital mammograms," Radiographics, 16(6), 1403-11 (1996).

[24] Byng, J. W., Critten, J. P., and Yaffe, M. J., “Thickness-equalization processing for mammographic images," Radiology, 203(2), 564-8 (1997).

[25] Karssemeijer, N., "Automated classification of parenchymal patterns in mammograms," Phys Med Biol, 43(2), 365-78 (1998).

[26] Otsu, N., "A Threshold Selection Method from Gray-Level Histograms," IEEE Transactions on Systems, Man, and Cybernetics, 9(1), 62-66 (1979).

[27] Precht, H., Tingberg, A., Waaler, D. et al., "New Developed DR Detector Performs Radiographs of Hand, Pelvic and Premature Chest Anatomies at a Lower Radiation Dose and/or a Higher Image Quality,” J Digit Imaging, (2013). 
[28] Sund, P., Bath, M., Kheddache, S. et al., "Comparison of visual grading analysis and determination of detective quantum efficiency for evaluating system performance in digital chest radiography," Eur Radiol, 14(1), 48-58 (2004). 